\title{
Fluid-physical simulation of silicate scale formation using lattice Boltzmann method
}

\author{
Akihiro MIZUSHIMA, Hitoshi MIKADA, Tada-nori GOTO and Junichi TAKEKAWA
}

Kyoto University

\begin{abstract}
Scaling is a key phenomenon that obstructs fluid circulation in plumbing system. Our goal in this study is to evaluate the importance of both chemical kinetic and hydrodynamic effects on silica deposition. For qualitative and quantitative discussion, we estimate the amount and the distribution of silica deposition with these two processes based on the numerical simulation and compare with the observation from a field experiment. We solve the fluid, temperature and dissolved silica concentration field by using the lattice Boltzmann method. From our simulation results, it is found that the effects of the hydrodynamic process are very important to reproduce the growth of scale qualitatively, whereas the simple chemical kinetic deposition could not sufficiently contribute to the real silica deposition. It is, therefore, necessary to emphasize the hydrodynamic effect should be take into account for reproducing silica scaling.
\end{abstract}

\section{INTRODUCTION}

The formation of inorganic sparingly-soluble salts, anhydrite and silica from brine is as a cause of scale in pipeline system. Microscopically, these materials could also be a cause of the cementation that affects the circulation of subsurface or sub-seafloor hydrothermal systems. Choking of flow channels are famous phenomena that reduce the efficiency in geothermal power plants. Silica can be extracted from the rock at depth by water-rock chemical reactions in the earth's crust and will be transported toward the surface in the hydrothermal solution. When hydrothermal fluid containing silica ascends toward the earth's surface and subjected to cooling, the solution become super-saturated with silica and precipitation will occur (Martin and Lowell, 2000). Although silica scaling is a widely observed problem in geosciences fields, the actual mechanism is not established.

There has been a lot of works emphasizing the simple reaction kinetics of silica and how they are affected by temperature and degree of super-saturation. (e.g. Rimstidt and Barnes, 1980). Although Rimstidt and Barnes (1980) determined the kinetic parameter using both published data and experimental one as a function of the temperature, it has been reported that the magnitude of deposition rate is extremely lower than that measured in the laboratories or in the fields (Malate and O'Sullivan, 1992; Weir and White, 1996). It can be reported that there was no difference in the amount of silica deposition before and after the fluid temperature reduction (Kawahara et al., 2013). As a high rate of deposition is observed where the flow stagnates, the inhomogeneous fluid flow could influence the scale growth significantly. Nevertheless, relatively little reports have been undertaken to investigate hydrodynamic effect on silica scaling. The aim of this work is to evaluate the contribution of the chemical kinetic or the hydrodynamic process to silica scaling by comparing the numerical result with data from a field observation. Our ultimate goal is to help us to understand the macroscopic flow in sub-seafloor hydrothermal systems by the feedback on the result of our channel-scale model.

\section{METHOD}

\section{(1) Lattice Boltzmann Method}

In this paper, we use the lattice Boltzmann method to simulate fluid flow. In the lattice Boltzmann model, the two-dimensional nine -directional (D2Q9) lattice Bhatnager-Gross-Krook model (LBGK) is adopted. In this model, the directions of the discrete velocity are given as

$$
\boldsymbol{c}_{i}=\left\{\begin{array}{cl}
c\{\cos (i \pi / 4), \sin (i \pi / 4)\} & \text { for } i=0,2,4,6 \\
\sqrt{2} c\{\cos (i \pi / 4), \sin (i \pi / 4)\} & \text { for } i=1,3,5,7 \\
(0,0) & \text { for } i=8
\end{array}\right.
$$

where $c=\Delta x / \Delta t$ is the particle speed. $\Delta x$ and $\Delta t$ are the lattice spacing and the time step, respectively. The evolution equation of the particle velocity distribution function $f_{i}(\boldsymbol{x}, t)$ leads

$f_{i}(\boldsymbol{x}+\Delta \boldsymbol{x}, t+\Delta t)-f_{i}(\boldsymbol{x}, t)=-\frac{1}{\tau}\left[f_{i}(\boldsymbol{x}, t)-f_{i}^{e q}(\boldsymbol{x}, t)\right]$,

where the relaxation time $\tau$ is a parameter which characterizes the constitutive behavior of the fluid 
at a macroscopic level. It is related to the macroscopic kinetic viscosity $v$ of the fluid as

$$
v=\frac{c^{2} \Delta x}{3}\left(\tau-\frac{1}{2}\right) .
$$

$f_{i}^{e q}(\boldsymbol{x}, t)$ is the local equilibrium distribution function. In order to develop the correct Navier-Stokes equations, the local equilibrium distribution function is defined as

$$
f_{i}^{e q}=\omega_{i} \rho\left[1+3 \frac{\left(\boldsymbol{c}_{i} \cdot \boldsymbol{u}\right)}{c^{2}}+\frac{9}{2} \frac{\left(\boldsymbol{c}_{i} \cdot \boldsymbol{u}\right)^{2}}{c^{4}}-\frac{3}{2} \frac{\boldsymbol{u}^{2}}{\boldsymbol{c}^{2}}\right],
$$

where $\omega_{i}$ represent the weight factors, $\rho$ is the density and $\boldsymbol{u}$ is the flow velocity vector given as follow:

$$
\rho=\sum_{i=0}^{8} f_{i}(\boldsymbol{x}, t), \boldsymbol{u}=\sum_{i=0}^{8} f_{i}(\boldsymbol{x}, t) \cdot \boldsymbol{c}_{i} .
$$

We describe the transport of the temperature and the concentration of the dissolved silica with the convection-diffusion equation (CDE) written as

$$
\frac{\partial C}{\partial t}+(\boldsymbol{u} \cdot \nabla) C=D \nabla^{2} C+Q
$$

where the macroscopic variable $C$ in the $\mathrm{CDE}$ is the temperature or the concentration. $D$ is the diffusion coefficient of the temperature or the concentration of the dissolved silica. $Q$ is the source term related to the decreased concentration of the dissolved silica due to the silica deposition in the concentration field. The evolution of the system is

$$
g_{i}(\boldsymbol{x}+\Delta \boldsymbol{x}, t+\Delta t)-g_{i}(\boldsymbol{x}, t)=-\frac{1}{\tau_{s}}\left[g_{i}(\boldsymbol{x}, t)-g_{i}^{e q}(\boldsymbol{x}, t)\right]+\omega_{i} \Delta t Q,
$$

where $g_{i}(\boldsymbol{x}, t)$ is the distribution function, $g_{i}^{e q}(\boldsymbol{x}, t)$ is the equilibrium distribution function and $\tau_{s}$ is the relaxation time. The relationship between $\tau_{s}$ and $D$ is

$$
D=\frac{2 c^{2} \rho}{3}\left(\tau_{g}-\frac{1}{2}\right) .
$$

In this paper, a D2Q9 model is used and the equilibrium distribution function $g_{i}^{e q}(\boldsymbol{x}, t)$ is taken as

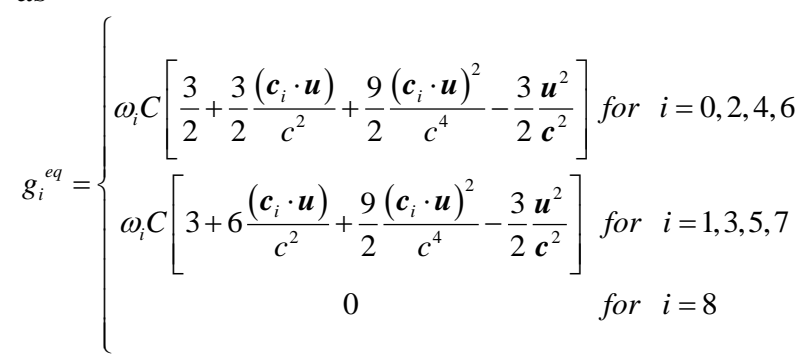

The temperature or the concentration of the dissolved silica $C$ is defined as

$$
C=\sum_{i=0}^{8} g_{i}
$$

\section{(2) Mathematical modeling of silica deposition}

In this paper, we consider the chemical kinetic and the hydrodynamic processes of the silica deposition.

In the chemical kinetic process, the overall reaction used to describe the silica precipitation is

$$
\mathrm{SiO}_{2}(s)+2 \mathrm{H}_{2} \mathrm{O} \rightleftharpoons \mathrm{Si}(\mathrm{OH})_{4} \text {. }
$$

In this paper the deposition rate law proposed by Rimstidt and Barnes (1980) is given by the expression:

$$
\frac{\partial R}{\partial t}=k_{+} M_{\mathrm{SiO}_{2}}\left(\frac{m_{\mathrm{Si}(\mathrm{OH})_{4}}}{K_{e q}}-1\right)
$$

where $R$ is the mass of the surface deposition par $\mathrm{m}^{2}$, the $t$ is time and $M_{\mathrm{SiO}_{2}}$ is the molecular weight of the silica deposition. $k_{+}$is the dissolution rate constant and $K_{e q}$ is the equilibrium constant. $m_{\mathrm{Si}(\mathrm{OH})_{4}}$ is the concentration of the dissolved silica. $k_{+}$and $K_{e q}$ is given respectively as a function of temperature by

$$
\begin{aligned}
& \log k_{+}=-0.369-7.890 \times 10^{-4} T-3438 / T, \\
& \log K=0.3380-7.889 \times 10^{-4} T-840.1 / T .
\end{aligned}
$$

where $T$ is in Kelvin.

In the hydrodynamic process, the deposition rate is expressed by

$$
\frac{\partial R}{\partial t}=\psi\left(S^{-0.630}\right) m_{\mathrm{Si}(\mathrm{OH})_{4}},
$$

where $S$ is the shear rate near the wall. $\psi$ is the coefficient determined according to Gentile et al. (2008). Gentile et al. (2008) formulated the relation between the shear rate near the wall and the number of the $1 \mu \mathrm{m}$ spherical silica particles adhered on the wall. We assume that the number of the dissolved silica adhered on the wall is equal to that of the $1 \mu \mathrm{m}$ spherical silica particles.

\section{SIMULATION MODEL}

In order to predict the location of silica deposition based on both the chemical kinetic and the hydrodynamic process, we use the 2D parallel plate channel model with the sudden expansion of the width (Figure 1). Mercado et al. (1989) showed the location of scale deposition on the geothermal 
production-pipe wall in a schematic cross section. Their observation can be as the reference.

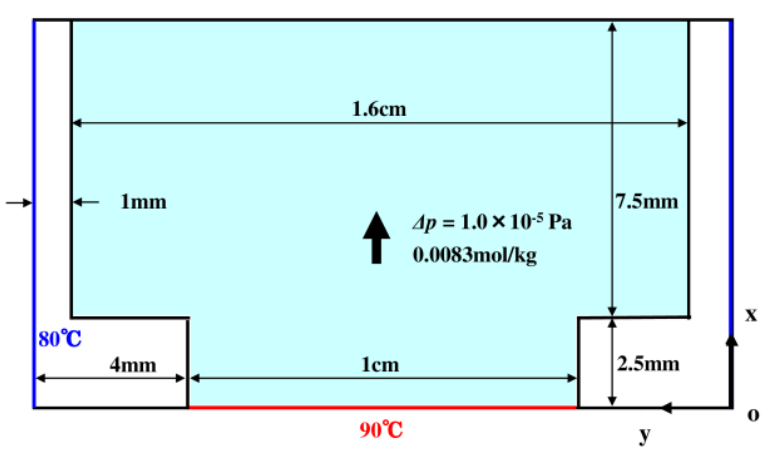

Figure 1 Model (initial condition)

\section{RESULT}

Figure 2(a), (b) and (c) show the distribution of the flow velocity and the pressure, the temperature and the dissolved silica concentration respectively obtained when the steady state is achieved. As shown in Figure 2(a), it is found that the flow stagnates in the corners of the sudden expansion of the width.

The gradual reduction slope of silica deposition along the wall surface is found based on the chemical kinetic process, while a high rate of silica deposition is found at the points which are the stagnation point and the corners of the sudden expansion of the width based on the hydrodynamic process. Therefore, the stagnation point of a sudden expansion of flow path at joint shows voluminous scaling predicted by not the chemical kinetic but the hydrodynamic process. It is found that the amount of silica deposition estimated by the hydrodynamic process is orders of magnitude larger than estimated by the chemical kinetic process. Figure 3 and $\mathbf{4}$ show the mass of silica deposition along the wall surface par $1 \mathrm{~m}^{2}$ estimated by the chemical kinetic and the hydrodynamic processes 200s after initiation of calculation.

It is found that the build-up of silica scale fills the recess of the points indicated by the yellow star (Figure 4) in which the flow stagnates and the gradual reduction slope of silica deposition is finally formed at the downstream-side wall of the sudden expansion. The silica scale distribution finally obtained by the hydrodynamic process is consistent with the observed in the vicinity of the casing shoe of production-wall pipe of the geothermal well (Mercado et al., 1989). Figure 5 shows the time evolution of the growth of silica scale. Since the growth rate of the silica scale is too slow, only the deposition rate is increased by a factor of $10^{5}$ in order to predict the long-term variation of the silica scale.

\section{DISCUSSION AND CONCLUSIONS}

We investigated the effect of the chemical kinetic and the hydrodynamic processes on the silica scale using a casing shoe model with the lattice Boltzmann method. It is found that the effect of the simple chemical kinetic process on the silica scale solely could not sufficiently explain the real silica deposition qualitatively. On the other hand, the pattern of the scale growth estimated by the hydrodynamic process becomes consistent with the real field. Since the amount of silica deposition estimated by the hydrodynamic process is extremely larger than estimated by the chemical kinetic process, the hydrodynamic effect are dominant among the effects on silica scaling in contrast to the simple chemical kinetic effect. Some works (Malate and O'Sullivan, 1992; Weir and White, 1996) suggested that the chemical kinetic model of silica precipitation (Rimstidt and Barnes, 1980) predicts about orders of the magnitude slower scale growth than the real field and adjusted kinetic parameters by fitting the model to the field data. We emphasize that the hydrodynamic effects should be take account of describing scaling phenomenon. 

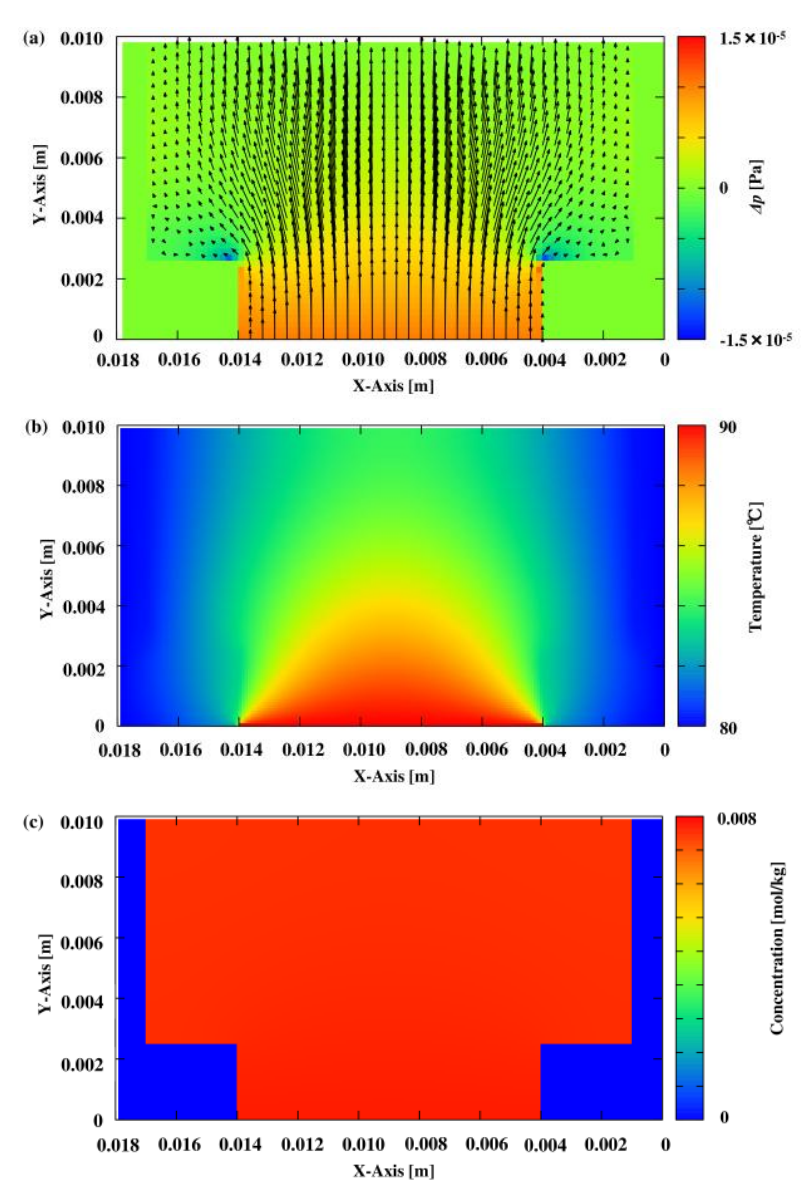

Figure 2 Distribution of (a) flow velocity and pressure, (b)temperature and (c) dissolved silica concentration; $t=200 \mathrm{~s}$.
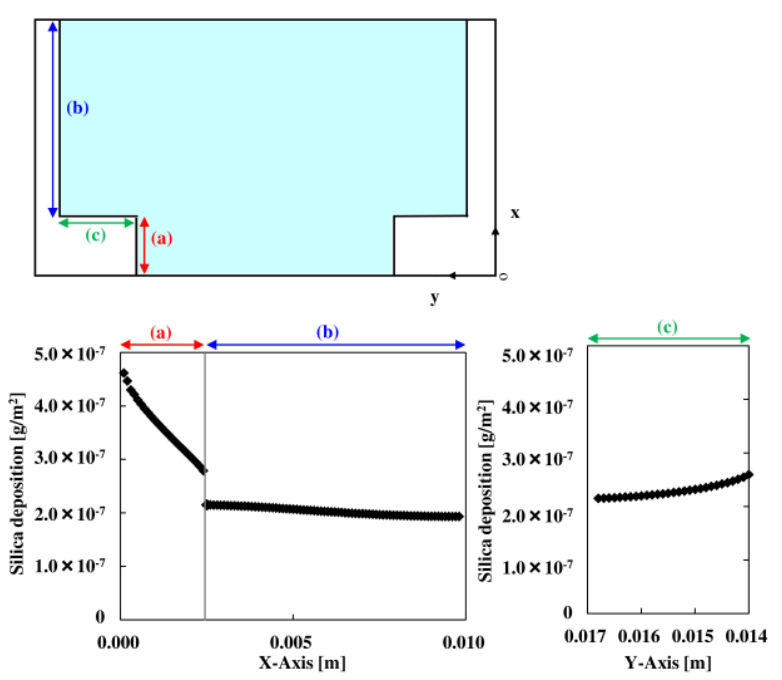

Figure 3 Mass of silica deposition along surface of vertical (a, b) and horizontal (c) wall estimated by chemical kinetic process.
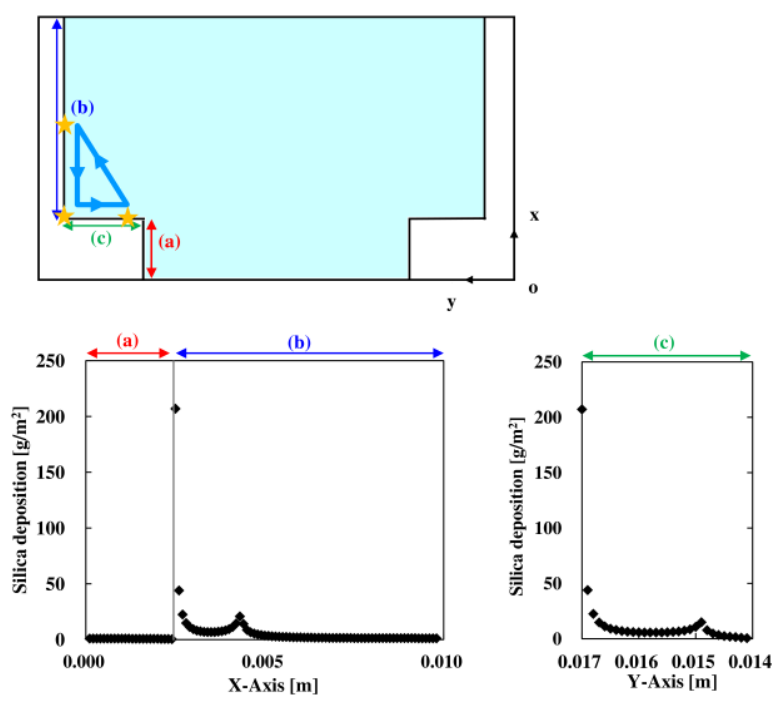

Figure 4 Mass of silica deposition along surface of vertical (a, b) and horizontal (c) wall estimated by hydrodynamic process.

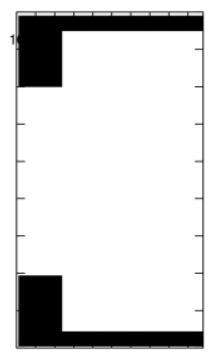

(a) $0 \mathrm{~s}$

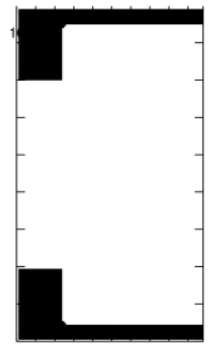

(b) $0.02 \mathrm{~s}$

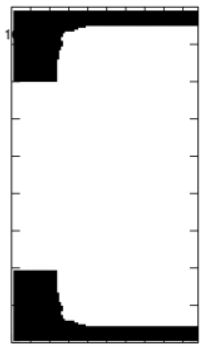

(c) $0.04 \mathrm{~s}$

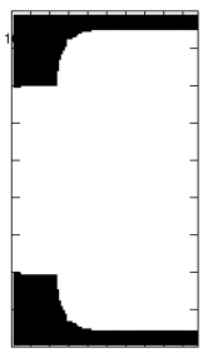

(d) $0.05 \mathrm{~s}$

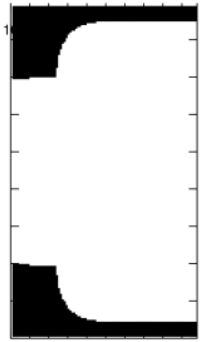

(e) $0.06 \mathrm{~s}$

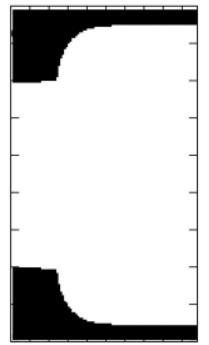

(f) $0.08 \mathrm{~s}$

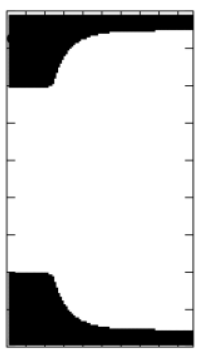

(g) $0.12 \mathrm{~s}$

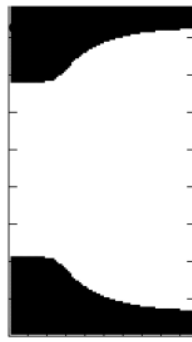

(h) $0.4 \mathrm{~s}$

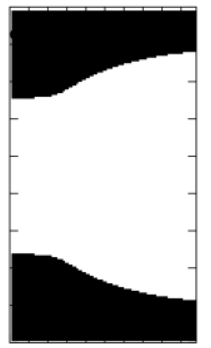

(i) $0.8 \mathrm{~s}$
Figure 5 Time change of build-up of silica scale (a) $0 \mathrm{~s}$, (b) $0.02 \mathrm{~s}$, (c) $0.04 \mathrm{~s}$, (d) $0.05 \mathrm{~s}$, (e) $0.06 \mathrm{~s}$, (f) $0.08 \mathrm{~s},(\mathrm{~g}) 0.12 \mathrm{~s},(\mathrm{~h}) 0.40 \mathrm{~s}$ and (i) $0.80 \mathrm{~s}$ after initial of calculation. 


\section{REFERENCES}

1) Gentile, F., Chiappini, C., Fine, D., Bhavane, R. C., Peluccio, M. S., Ming-Cheng Cheng, M., Liu, X. and Decuzzi, P., 2008, The effect of shape on the margination dynamics of non-neutrally buoyant particles in two-dimensional shear flows, Journal of Biomechanics, 41, 2312-2318.

2) Hosoi, M. and Imai, H., 1982, Study on precipitation and prevention of the silica scale form the geothermal hot water, Journal of the Geothermal Research Society of Japan, 4, 127-142.

3) Malate, R. C. M. and O'Sullivan, 1992, Mathematical modeling of silica deposition in a porous medium, Geothermics, 21, no. 3, 377-400.

4) Martin, T. and Lowell, P., 2000, Precipitation of quartz during high-temperature, fracture-controlled hydrothermal upflow at ocean ridges: Equilibrium versus linear kinetics: Journal of Geophysical Research, 105, no. B1, 869-882.

5) Mercado, M., Bermejo, F., Hurtado, R., Terrazas, B. and Hernandez, L., 1989, Scale incidence of production pipes of Cerro Prieto geothermal wells: Geothermics, 18, no. 1/2, 225-232.

6) Rimstidt, J. D. and Barnes, H. L., 1980, The kinetics of silica-water reactions: Geochimica et Cosmochimica Acta, 44, 1683-1699.

7) Weir, G. J. and White, S. P., 1996, Surface deposition from fluid flow in a porous medium, Transport in Porous Media, 25, 79-96. 